\title{
Fatores Relacionados às Ocorrências Éticas na Enfermagem Frente a Erros de Medicação
} Factors Related to Ethical Occurrences in Nursing Concerning Medication Errors

\author{
DEYLA MOURA RAMOS ISOLDI ${ }^{1}$ \\ MARILUCI CAMARGO FERREIRADA SILVA CANDIDO² \\ CLÉLIAALBINO SIMPSON ${ }^{3}$ \\ FERNANDO SOUZA SILVA ${ }^{4}$
}

\section{RESUMO}

Objetivo: Objetiva-se identificar os fatores determinantes para as ocorrências éticas que envolvem erro de medicação na enfermagem. Material e Métodos: Trata-se de um estudo do tipo revisão da literatura que buscou avaliar a literatura existente sobre as ocorrências éticas relacionadas a erros medicamentosos na enfermagem. Para o levantamento bibliográfico feito em 2014, realizou-se a busca em duas bases de dados eletrônicas Literatura Latino-Americana e do Caribe em Ciências da Saúde e no Scientific Electronic Library Online, utilizando os descritores: Ética de enfermagem, erros de medicação, equipe de enfermagem, Enfermagem e ética. Selecionaram-se artigos disponíveis eletronicamente e que respondessem ao objetivo proposto. Resultados: Foram captados 31 trabalhos. Destes, seis não estavam disponíveis no meio eletrônico e três se repetiam. Após leitura dos textos completos, dez não possuíam relação com a temática, com isso a amostra final foi de 13 trabalhos, todos artigos científicos. Os resultados mostram que os principais fatores relacionados às ocorrências éticas na enfermagem referiam-se às relações profissionais conflituosas e às iatrogenias, com ênfase a terapia medicamentosa. Conclusão: Os erros medicamentosos cometidos pelos profissionais de enfermagem estão associados com a negligência, imperícia e imprudência e os princípios bioéticos violados são os da não maleficência e o da beneficência.

\section{DESCRITORES}

Ética em Enfermagem. Erros de Medicação. Equipe de Enfermagem. Enfermagem. Ética.

\begin{abstract}
Objective: To identify the determining factors for ethical occurrences involving medication errors in nursing. Material and Methods: This was a literature review on the ethical occurrences related to medication errors in nursing. Bibliographical searches were carried out in 2014 in the databases Latin American and Caribbean Literature in Health Sciences and Scientific Electronic Library Online, using the descriptors: Nursing ethics, medication errors, nursing team, nursing and ethics. Electronic articles meeting the eligibility criteria were selected. Results: A total of 31 articles were retrieved. Of these, six were not available electronically and three were repeated entries. After reading the full texts, ten articles were found to be out of the scope, hence the final sample was composed of 13 scientific articles. The results show that the main factors related to ethical occurrences in nursing referred to conflicting professional relationships and iatrogeny, with emphasis on drug therapy. Conclusion: The medical errors committed by nursing professionals are associated with negligence, malpractice and recklessness, and the bioethical principles violated are those of nonmaleficence and beneficence.
\end{abstract}

\section{DESCRIPTORS}

Nursing Ethics. Medication Errors. Nursing Team. Nursing. Ethic.

1 Doutoranda em Enfermagem pelo Programa de Pós-Graduação em Enfermagem, Universidade Federal do Rio Grande do Norte. Natal, RN, Brasil. Docente da Faculdade Maurício de Nassau. Parnamirim. Rio Grande do Norte. Brasil.

2 Docente do Departamento de Enfermagem e do Programa de Pós-Graduação em Enfermagem (Mestrado Acadêmico e Doutorado) da Universidade Federal de Mato Grosso do Sul-UFMS. Mato Grosso do Sul. Brasil.

3 Docente Associado, do Departamento de Enfermagem e do Programa de Pós-Graduação em Enfermagem (Mestrado Acadêmico e Doutorado) da Universidade Federal do Rio Grande do Norte-UFRN. Natal. Rio Grande do Norte. Brasil.

4 Enfermeiro do Hospital Universitário Onofre Lopes - HUOL. Docente da Universidade Estácio de Sá. Natal. Rio Grande do Norte. Brasil. 
A s ocorrências éticas envolvendo os erros de medicações administrados pelas equipes de nfermagem, têm se transformado em uma constante nos noticiários, fato que nos preocupa pela relevância que o tema possui para a profissão, a sociedade e a saúde pública.

Os eventos éticos causam prejuízos ou danos aos pacientes e aos profissionais de saúde, e estão intimamente relacionados à falta de atenção, de destreza, de habilidade ou de conhecimento técnico para execução de um determinado procedimento de enfermagem, além de imprudência, a qual se caracteriza pela atitude precipitada no momento da ação, seja em relação ao paciente, nas relações interpessoais ou interprofissionais, por exemplo, quando essas relações se revelam desrespeitosas ${ }^{1}$.

Dentre os fatores que colaboram para o aumento dos erros está o crescente número de medicamentos lançados no mercado, exigindo dos profissionais constantes atualizações, bem como a exigência de maior complexidade na terapêutica ${ }^{2}$. O conhecimento dos novos medicamentos é considerado uma boa estratégia para a redução de erro.

É responsabilidade da equipe de enfermagem manter o cliente livre de danos decorrentes de imperícia, negligência ou imprudência. A negligência consiste na falta de atenção ou inação; a imperícia decorre da inabilidade ou deficiente destreza; a imprudência caracteriza-se por uma atitude precipitada ${ }^{1}$

As ocorrências éticas podem ser decorrentes da negligência, imperícia ou imprudência, podendo expor o paciente a situações de riscos, mesmo que não seja a intenção dos profissionais de ocasionar esses danos ao cliente ${ }^{3}$.

A prestação de assistência à saúde isenta de riscos e falhas ao paciente, mais do que um objetivo a ser atingido por todos os profissionais da área de saúde, é um compromisso da própria formação profissional.

Destarte, os profissionais de enfermagem devem possuir conhecimento técnico e buscar atualização constante, compromisso ético para impedir as ocorrências danosas ao cliente. Assim, os profissionais precisam entender sobre responsabilidade ética, profissional, civil e penal de suas ações não excluindo seus direitos e deveres, para evitar negligência, Imperícia ou imprudência 4 .

Nesse sentido, o Código de Ética dos Profissionais de Enfermagem em seu artigo 113 define infração ética como a "ação, omissão ou conivência que implique em desobediência e/ou inobservância às ações desse código"5. Geralmente, quando um profissional age de forma culposa, ele não almeja causar um malefício a alguém, no entanto, devido à sua falta de atenção, de conhecimento ou imprudência, o seu agir poderá resultar em riscos, os quais podem ser evitados.

A Lei do Exercício Profissional de Enfermagem n. ${ }^{\circ} 7.498$, de 25 de junho de 1986, estabelece as competências dos profissionais de enfermagem e se constitui uma forma de se aferir a responsabilidade no agir com base nas competências técnicas, éticas, políticas ou relacionais de cada um. De acordo com a legislação vigente, cabe ao enfermeiro o gerenciamento das ações, por meio da sistematização da assistência, ao planejar, executar, avaliar e discutir os resultados das condutas propostas com a sua equipe ${ }^{1}$.

O enfrentamento, e especialmente a prevenção das ocorrências éticas, representam um constante desafio para o enfermeiro. Portanto, os conhecimentos adquiridos e as experiências vividas revelam importantes motivos do porque os profissionais agem nas ocorrências éticas de enfermagem. Essa dimensão permeia as relações interpessoais, não somente no que tange aos direitos do paciente, quanto no convívio com os demais profissionais da equipe de trabalho ${ }^{6}$.

Tem-se observado uma maior colaboração dos profissionais em empresas que promovem suporte e procuram compreender os motivos que levaram aos erros, quando comparadas às instituições que penalizam os funcionários cometedores de infrações. Os serviços de saúde vêm buscando atingir níveis cada vez mais altos de excelência no atendimento, visando proporcionar assistência livre de riscos e danos ao paciente ${ }^{7}$.

Tratar das ocorrências éticas relacionadas somente à questão da formação profissional seria muito ingênuo e uma abordagem simplista para contemplar uma situação dessa complexidade que na prática profissional envolve múltiplos fatores.

Como já foi dito, tal problemática abrange diferentes fatores relacionados às ocorrências éticas, e por esse motivo resolveu-se investigá-las.

Este estudo justifica-se pela relevância que a temática tem para a enfermagem e pela necessidade observada em prática profissional, na qual a abordagem equivocada da ética, envolvendo os erros de medicação, consiste em uma constante cotidiana no cuidado.

Diante da problemática apresentada, elaboramos o seguinte questionamento: $O$ que se tem publicado em âmbito nacional e internacional a respeito das ocorrências éticas cometidas pelos profissionais da enfermagem? Desse modo, 
objetivou-se identificar os fatores determinantes para as ocorrências éticas que envolvem erro de medicação na enfermagem.

\section{MATERIAL E MÉTODOS}

Trata-se de estudo do tipo revisão integrativa, que buscou avaliar a literatura existente para fornecimento de subsídios à análise dos artigos sobre ocorrências éticas envolvendo erros de medicação na prática da enfermagem. Para elaboração deste estudo foram observadas as seguintes fases: a) elaboração do protocolo do estudo; b) busca dos manuscritos publicados em periódicos; c) categorização dos estudos; d) avaliação dos artigos incluídos na revisão integrativa; e) interpretação dos resultados e síntese do conhecimento evidenciado nos artigos analisados ${ }^{8}$. O protocolo do estudo contém a questão de pesquisa, o objetivo proposto, estratégias de busca, seleção dos estudos e coleta dos dados.

Realizou-se uma busca por meio eletrônico em 2014, utilizando-se o vocabulário estruturado Decs - Descritores em Ciências da Saúde, e subsequente pesquisa via formulário na base de dados Literatura Latino-Americana e do Caribe em Ciências da Saúde - Lilacs por descritor de assunto e índice, e no Scientific Electronic Library Online SciELO. O acesso em cada base de dados foi efetuado em um único dia.

Para a busca dos artigos, estabeleceu-se os seguin-tes descritores: "Ética em enfermagem", "Erros de medicação", "Equipe de enfermagem", "Enfermagem" e "Ética". A seguir foram feitos 13 cruzamentos associando os descritores mencionados. Dos estudos captados nestes cruzamentos foram excluídas as repetições, assim como os descritores que mostraram resultado nulo.

Dessa forma, buscou-se publicações que apresentassem esses descritores no título, resumo ou nas próprias palavras-chave dos artigos disponíveis nas bases de dados especificadas respeitando os critérios de inclusão e exclusão.

Os critérios de inclusão definidos para a seleção dos artigos foram: artigos com abordagem da temática proposta em português e inglês; manuscritos publicados na íntegra e disponíveis eletronicamente, por favorecer o processo de avaliação dos mesmos; pesquisas de acesso livre. Não foram estabelecidos periodicidade de publicação para não limitar os resultados.

Os critérios de exclusão para a presente proposta de estudo foram: artigos repetidos em mais de uma base de dados, os quais foram considerados somente uma vez, os editoriais, e os estudos que apesar de apresentarem os termos selecionados, não respondiam à questão norteadora.

Inicialmente a seleção dos artigos foi realizada mediante o atendimento dos critérios de inclusão e leitura prévia dos resumos, a fim de confirmar a temática proposta. Em caso de dúvidas sobre os discursos textuais dos estudos realizouse uma segunda análise do estudo onde decidiuse a sua inclusão para o tratamento dos dados.

Para organização e extração dos dados dos estudos elaborou-se um quadro para captar informações referentes à identificação do artigo, origem, idioma, ano de publicação, dados dos autores, título do trabalho, população estudada, objetivo, tipo de ocorrência e/ou infração ética, descrição metodológica, e principais resultados.

Nessa busca, encontrou-se 31 artigos nas bases de dados pesquisadas, destes, 6 não estavam disponíveis em meio eletrônico e três se repetiam. Após a leitura dos textos completos quais foram salvos os títulos e os resumos em documento do Word para evitar que, ao acessar em outro dia, ocorresse mudança na quantidade de artigos. Em seguida, foram lidos todos os títulos e resumos para saber quais artigos atendiam aos critérios de inclusão.

Após a seleção, excluíram-se aqueles que não abordavam a temática de modo a alcançar o objetivo do estudo. Assim, selecionou-se um total de 13 artigos. A pesquisa não foi submetida ao Comitê de Ética em Pesquisa pelo fato de tratar de uma revisão com busca apenas na literatura.

\section{RESULTADOS E DISCUSSÃO}

Foram analisadas 13 publicações que subsidiaram a identificação das ocorrências éticas na enfermagem, conforme Quadro 1.

Os cruzamentos com os descritores foram: enfermagem, ética em enfermagem e erros de medicação; enfermagem e ética em enfermagem; enfermagem, erros de medicação e equipe de enfermagem; enfermagem, equipe de enfermagem e ética; enfermagem e erros de medicação; ética em enfermagem, erros de medicação e equipe de enfermagem; ética em enfermagem, equipe de enfermagem e ética; erros de medicação, equipe de enfermagem e ética; ética, enfermagem e erros de medicação; erros de medicação e ética; equipe de enfermagem e ética; enfermagem e ética; ética, enfermagem e ética em enfermagem, todos os cruzamentos acrescido da expressão booleano AND.

A área da enfermagem apresentou uma 


\begin{tabular}{|c|c|c|c|}
\hline \multicolumn{4}{|c|}{$\begin{array}{l}\text { Quadro 1. Síntese dos estudos selecionados ( } n=13 \text { ), segundo autores, ano de publicação, periódico e título, } \\
\text { Natal, } 2014 .\end{array}$} \\
\hline Artiqo & Autores & Periódico & Título \\
\hline $\mathrm{A} 1$ & Freitas GF, Oguisso T. 2008 & $\begin{array}{l}\text { Rev Esc Enferm } \\
\text { USP. }\end{array}$ & $\begin{array}{l}\text { Ocorrências éticas com profissionais de } \\
\text { enfermagem: um estudo quantitativo. }\end{array}$ \\
\hline $\mathrm{A} 2$ & Melo ABR, Silva LD. 2008 & $\begin{array}{l}\text { Esc Anna Nery Rev } \\
\text { Enferm. }\end{array}$ & $\begin{array}{l}\text { Segurança na terapia medicamentosa: uma revisão } \\
\text { bibliográfica }\end{array}$ \\
\hline A3 & $\begin{array}{l}\text { Belela ASC, Peterlini MAS, } \\
\text { Pedreira MLG. } 2010\end{array}$ & $\begin{array}{l}\text { Rev Bras Ter } \\
\text { Intensiva. }\end{array}$ & $\begin{array}{l}\text { Revelação da ocorrência de erro de medicação em } \\
\text { unidade de cuidados intensivos pediátricos. }\end{array}$ \\
\hline A4 & Pelliciotti JSS, Kimura M. 2010 & $\begin{array}{l}\text { Rev. Latino-Am. } \\
\text { Enfermagem. }\end{array}$ & $\begin{array}{l}\text { Erros de medicação e qualidade de vida } \\
\text { relacionada à saúde de profissionais de } \\
\text { enfermagem em unidades de terapia intensiva }\end{array}$ \\
\hline A5 & $\begin{array}{l}\text { Yamamoto MS, Peterlini MAS, } \\
\text { Bohomol E. } 2011\end{array}$ & Acta Paul Enferm & $\begin{array}{l}\text { Notificação espontânea de erros de medicação em } \\
\text { hospital universitário pediátrico }\end{array}$ \\
\hline A6 & Camerinil FG, Silva LD. 2011 & $\begin{array}{l}\text { Texto Contexto } \\
\text { Enferm }\end{array}$ & $\begin{array}{l}\text { Segurança do paciente: análise do preparo de } \\
\text { medicação intravenosa em hospital da rede } \\
\text { sentinela. }\end{array}$ \\
\hline A7 & Bohomol E, Ramos LH. 2007 & Rev Bras Enferm & $\begin{array}{l}\text { Erro de medicação: importância da notificação no } \\
\text { qerenciamento da sequrança do paciente. }\end{array}$ \\
\hline A8 & Franco JN. et al., 2010 & $\begin{array}{l}\text { Rev Bras } \\
\text { Enfermagem }\end{array}$ & $\begin{array}{l}\text { Percepção da equipe de enfermagem sobre fatores } \\
\text { causais de erros na administração de } \\
\text { medicamentos. }\end{array}$ \\
\hline A9 & Silva AEBC. et al., 2007 & Acta Paul Enferm & $\begin{array}{l}\text { Problemas na comunicação: uma possível causa } \\
\text { de erros de medicacão. }\end{array}$ \\
\hline A10 & Paula GS. et al., 2010 & Rev Chía. Colombia & $\begin{array}{l}\text { O sofrimento psiquico do profissional de } \\
\text { enfermagem da unidade hospitalar. }\end{array}$ \\
\hline A11 & Bustos MZ.et al., 2008 & $\begin{array}{l}\text { Brazilian Journal of } \\
\text { Pharmaceutical } \\
\text { Sciences }\end{array}$ & $\begin{array}{l}\text { Prescription errors in a primary care university unit: } \\
\text { urgency of pharmaceutical care in Mexico. }\end{array}$ \\
\hline A12 & $\begin{array}{l}\text { Schneider DG, Ramos FRS. } \\
\qquad 2012\end{array}$ & $\begin{array}{l}\text { Rev. Latino-Am. } \\
\text { Enfermagem }\end{array}$ & $\begin{array}{l}\text { Processos éticos de enfermagem no Estado de } \\
\text { Santa Catarina: caracterização de elementos } \\
\text { fáticos. }\end{array}$ \\
\hline A13 & Freitas GF, Oguisso T. 2007 & Acta Paul Enferm & $\begin{array}{l}\text { Perfil de profissionais de enfermagem e } \\
\text { ocorrências éticas }\end{array}$ \\
\hline
\end{tabular}

totalidade de $100 \%$ nas publicações e os estudos utilizaram na maioria, pesquisa de campo com abordagem qualitativa. Das 13 publicações estudadas, todas possuem palavras-chave, as quais são: violência, formação ética, enfermagem, erros de medicação, qualidade, gerenciamento, sistemas de medicação no hospital, comunicação, hospitalização, medida de segurança, administração hospitalar e controle de riscos. As palavras-chave mais utilizadas foram: enfermagem e erros de medicação.

Informações a respeito dos objetivos são ilustrados no quadro 2.

As dificuldades para os relatos dos erros de medicação prejudicam a avaliação dos tipos e do número de erros registrados e, consequentemente, não são documentados o número real de ocorrências. A subnotificação dos registros de erros está relacionada exclusivamente quando são gerados danos aos pacientes ${ }^{9}$.

Além disso, o medo de punições, da demissão, e do julgamento que pode ser atribuído ao profissional, tem sido determinante para a opção de não divulgar o fato, esquecendo-se do malefício que esse ato pode trazer ao paciente ${ }^{9}$.

Um estudo demonstrou que $87,3 \%$ da equipe de enfermagem têm conhecimento da necessidade de se notificar um erro de medicação e que $70,1 \%$ afirmam que as razões para a não notificação dos erros estão relacionadas ao medo da reação dos enfermeiros responsáveis ou colegas de trabalho ${ }^{10}$.

Assim, superar as falhas e problemas requer o conhecimento de que as atividades de assistência à saúde possuem fragilidades que podem comprometer a segurança do paciente ${ }^{11}$. Portanto, os profissionais de saúde precisam entender que relatar a infração cometida pode ser uma estratégia de prevenção.

Comumente adota-se a punição para os profissionais que cometem os erros de medicação, ao invés do apoio e entendimento dos motivos que levaram ao erro. Não é comum a priorização da abor-dagem de educação que possibilite a diminuição dos fatores de risco relacionados às infrações éticas na enfermagem ${ }^{10}$.

Na ocorrência de um erro, o enfermeiro deve iniciar uma investigação criteriosa evidenciando todos os detalhes, tais como, horário, pessoal envolvido, turno, tipo de erro, estágio do processo que ocorreu e possíveis falhas no sistema a fim de corrigir e preveni-los, e não atribuir a culpa somente ao profissional envolvido ${ }^{12}$. O enfermeiro deve aproveitar este momento e identificar as dificuldades do profissional e iniciar imediatamente orientação e treinamento.

Sabe-se que o trabalho educativo e não punitivo, em relação ao profissional de enfermagem que comete uma ocorrência ética, é a melhor forma 


\begin{tabular}{|c|c|}
\hline \multicolumn{2}{|r|}{$\begin{array}{l}\text { Quadro 2. Objetivos dos artigos selecionados ( } n=13) \text { com relação às ocorrências éticas de } \\
\text { enfermaqem, Natal, } 2014\end{array}$} \\
\hline Artigo & \\
\hline $\mathrm{A} 1$ & $\begin{array}{l}\text { Identificar as características das ocorrências éticas, envolvendo a assistência de enfermagem, em um hos } \\
\text { particular de qrande porte do município de São Paulo. }\end{array}$ \\
\hline A2 & sações científicas de enfermeiros sobre erros no manejo da terapia medicamentosa realizada \\
\hline A3 & $\begin{array}{l}\text { ência de comunicação do erro de medicação à equipe e família em uma unidade de cuidados } \\
\text { cos para atendimento de pacientes oncolóqicos. }\end{array}$ \\
\hline A4 & los por \\
\hline A5 & \\
\hline$A$ & \\
\hline A7 & Verificar \\
\hline A & \\
\hline A & 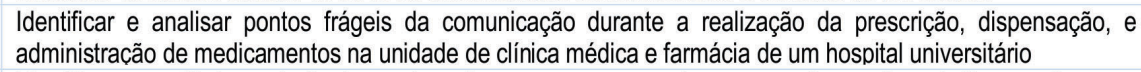 \\
\hline $\mathrm{A} 10$ & $\begin{array}{l}\text { rizar as condições de trabalho que levem } 0 \\
\text { síquico }\end{array}$ \\
\hline $\mathrm{A} 1$ & $\begin{array}{l}\text { Avaliar a racionalidade da prescrição e os erros de prescrição mais comuns na clínica de cuidados primários de } \\
\text { uma universidade mexicana. }\end{array}$ \\
\hline $\mathrm{A} 12$ & $\begin{array}{l}\text { Caracterizar os processos ético-profissionais de enfermagem, tramitados no Conselho Regional de } \\
\text { Enfermagem de Santa Catarina (Coren/SC), em seus elementos fáticos (ocorrências/infrações, causas e } \\
\text { desfechos). }\end{array}$ \\
\hline $\mathrm{A} 13$ & $\begin{array}{l}\text { Descrever o perfil dos profissionais de enfermagem envolvidos em ocorrências éticas de um determin } \\
\text { hospital. }\end{array}$ \\
\hline
\end{tabular}

gerencial a se seguir.

Um dos fatores determinantes é o problema na comunicação entre a equipe que se destaca como uma possível causa de erros na enfermagem. Assim, o sistema de medicação das instituições de saúde requer um processo de comunicação eficaz, estruturado de maneira a promover condições que auxiliem os profissionais na prevenção, para assegurar que o tratamento medicamentoso seja cumprido de forma segura ${ }^{13}$.

As prescrições verbais juntamente com cálculos errados de medicação, nomes similares, abreviação agrupado com distração, a pressa em atender os pacientes, desconhecimento da medicação e prescrição ilegível também são citadas como fatores que levam à ocorrência do erro ${ }^{12}$.

Outro problema observado foi o fato da equipe de enfermagem administrar o medicamento sem informar o paciente quanto aos riscos, custos e benefícios propostos destacando o desconhecimento sobre os efeitos medicamentosos ${ }^{12}$.

As iatrogenias, tanto as medicamentosas quanto as não medicamentosas, aparecem também como fator/ocorrência que estão associadas à imprudência, imperícia e negligência ${ }^{4}$.

Fatores como salários incompatíveis com o nível de responsabilidade dos profissionais, estresse, a grande variedade de procedimentos realizados, condições de insalubridade do ambiente laboral, a hierarquização, o ritmo e as longas jornadas de trabalho contribuem significativamente para uma assistência de risco ${ }^{14,15}$. Todos esses fatores são relevantes e merecem atenção ${ }^{12}$.

Nessa ótica, três hipóteses são elencadas como motivos para que ocorrências éticas possam acontecer no processo de cuidar: quando o profissional está sujeito a uma sobrecarga de trabalho maior do que é capaz de processar, quando existe uma queda ou baixo desempenho profissional do indivíduo que presta assistência direta ao paciente ou quando existe a combinação de fatores ${ }^{1}$.

Alternativas como as prescrições eletrônicas melhoraram a segurança do processo de prescrever e evita erros, devendo conter, obrigatoriamente a dose, a via e a frequência, devem ser legíveis, identificando aquele que a fez a todo o momento, informações referentes ao paciente devem ser fornecidas ao médico durante o processo e durante a prescrição devem ser detectados fatores que possam predispor o paciente a risco de reações adversas $^{16}$.

$\mathrm{O}$ agir do enfermeiro no processo de administração de medicamentos não é solitário, decorre de uma integração entre médicos, farmacêuticos, desenvolvendo um trabalho em equipe e objetivando os benefícios aos clientes ${ }^{10}$.

A própria natureza do tema e os riscos das implicações ético-legais levam a uma natural 
relutância em abordar o assunto, fazendo com que, dentro do hospital, as infrações éticas, não sejam monitoradas e discutidas.

Assim, a formação de grupos de discussão entre a enfermagem, melhorias do ambiente de trabalho, utilização das prescrições durante o preparo e administração, colocação dos nomes dos pacientes nos medicamentos preparados, cursos e treinamentos contínuos, são sugestões para maior qualidade e segurança na assistência aos pacientes, e implantação da prescrição por sistema computadorizado ${ }^{17}$.

Acredita-se que o incentivo para a notificação dos erros, a monitorização das ocorrências e fatores a elas relacionados, bem como ações menos punitivas e mais educativas venham favorecer a diminuição dos erros que tantos malefícios causam aos pacientes e a todos os envolvidos na sua assistência ${ }^{18}$.

Verificou-se que, devemos ficar alertas a estes indicadores, descobrir falhas, discutir, planejar, promover melhorias através da construção dos sistemas de segurança para prevenção de acidentes no processo de medicação.

Para que as ocorrências éticas sejam minimizadas e evitadas, acredita-se que o seu enfrentamento não será eficiente se não envolver

\section{REFERÊNCIAS}

1. Freitas GF, Oguisso T. Ocorrências éticas com profissionais de enfermagem: um estudo quantitativo. Rev Esc Enferm USP. 2008; 42(1):34-40

2. Melo ABR, Silva LD. Segurança na terapia medicamentosa: uma revisão bibliográfica. Esc Anna Nery Rev Enferm. 2008 mar; 12(1):166 - 72

3. Freitas GF, Oguisso T. Perfil de profissionais de enfermagem e ocorrências éticas. Acta Paul Enferm 2007;20(4):489-94.

4. Schneider DG, Ramos FRS. Processos éticos de enfermagem no Estado de Santa Catarina: caracterização de elementos fáticos. Rev. Latino-Am. Enfermagem jul.-ago. 2012; 20(4):744-52.

5. Conselho Federal de Enfermagem (COFEN). Resolução COFEN N. 240/2000. Aprova o Código de Ética dos Profissionais de Enfermagem e dá outras providências. In: Conselho Regional de Enfermagem de São Paulo (CORENSP). Documentos básicos de enfermagem: enfermeiros, técnicos e auxiliares. São Paulo; 2001.

6. Belela ASC, Peterlini MAS, Pedreira MLG. Revelação da ocorrência de erro de medicação em unidade de cuidados intensivos pediátricos. Rev Bras Ter Intensiva. 2010; 22(3):257-263

7. Pelliciotti JSS, Kimura M. Erros de medicação e qualidade de vida relacionada à saúde de profissionais de enfermagem em unidades de terapia intensiva. Rev. Latino-Am. Enfermagem. 2010; 18(6):1062-9.

8. Barros AJS, Lehfeld NAS. Fundamentos de Metodologia Científica. 3 ed. São Paulo: Pearson Prentice Hall, 2007.

9. Yamamoto MS, Peterlini MAS, Bohomol E. Notificação espontânea de erros de medicação em hospital universitário pediátrico. Acta Paul Enferm 2011; 24(6):766-71.

10. Bohomol E, Ramos LH. Erro de medicação: importância da notificação no gerenciamento da segurança do paciente. Rev Bras Enferm, Brasília 2007; 60(1):32-6.

11. Camerinil FG, Silva LD. Segurança do paciente: análise do preparo de medicação intravenosa em hospital da rede sentinela. Texto Contexto Enferm, Florianópolis, 2011; 20(1):41-9. ações que precisam acontecer desde a formação profissional na área de enfermagem e se estender durante toda a vida profissional.

Convém ressaltar a necessidade de educação em serviço de modo permanente para os profissionais em atividade, de forma que possam obter novos conhecimentos e estejam sempre reconstruindo o já aprendido a partir dos avanços nas várias esferas do conhecimento, estando, assim, capacitados para o exercício profissional em suas várias dimensões e despertando o interesse no sentido de prevenir situações que envolvem risco à segurança do paciente.

\section{CONCLUSÃO}

Por meio deste estudo conseguimos identificar que a execução do trabalho sob condições adversas, como disposição inadequada do ambiente, calor, sobrecarga de horários, tensões psicológicas extremas, estresse, a falta de comunicação entre as equipes, desconhecimento da medicação, pressa no atendimento e hierarquização são fatores relacionados às ocorrências éticas que podem levar o profissional da enfermagem a cometer erros medicamentosos.

12. Franco JN, Ribeiro G, D'innocenzo M, Barros BPA. Percepção da equipe de enfermagem sobre fatores causais de erros na administração de medicamentos. Rev Bras Enfermagem. 2010; 63 (6):927-32.

13. Silva AEBC, Cassiani SHB, Miasso Al, Opitz SP. Problemas na comunicação: uma possível causa de erros de medicação. Acta Paul Enferm. 2007; 20 (3):272-6.

14. Paula GS, Reis JF, Dias LC, Dutra VFD, Braga ALS, Cortez EA. O sofrimento psíquico do profissional de enfermagem da unidade hospitalar. Rev Chía. Colombia. 2010;10(3):267-79.

15. Telles Filho PCP, Praxedes MFS, Pinheiro MLP. Erros de medicação: análise do conhecimento da equipe de enfermagem de uma instituição hospitalar. Rev Gaúcha Enferm., Porto Alegre (RS) 2011; 32(3):539-45.

16. Bustos MZ, Castro-Pastrana LI, Reyes-Hernández I, LópezLuna MA, Bermúdez-Camps IB. Prescription errors in a primary care university unit: urgency of pharmaceutical care in Mexico. Revista Brasileira de Ciências Farmacêuticas. 2008; 44(1):115-25.

17. Miasso Al, Silva AEBC, Cassiani SHB, Grou CR, Oliveira RC, Fakih FT. O processo de preparo e administração de medicamentos: identificação de problemas para propor melhorias e prevenir erros de medicação. Rev Latino-am Enfermagem 2006; 14(3):354-63.

18. Padilha KG, Kitahara PH, Gonçalves CCS, Sanches ALC. Ocorrências iatrogênicas com medicação em Unidade de Terapia Intensiva: condutas adotadas e sentimentos expressos pelos enfermeiros. Rev Esc Enferm USP 2002; 36(1):50-7.

CORRESPONDÊNCIA

Deyla Moura Ramos Isoldi

Av. Brigadeiro Everaldo Breves, 64, Centro.

H30, BI 7 Casa 3.

Parnamirim - RN, Brasil - 59140-200

E-mail: deylaisoldi@gmail.com 